\title{
Vom Kampfblatt zum Flaggschiff
}

\author{
Eberhard Wolff \\ Prof. Dr. rer. soc., Redaktor Kultur, Geschichte, Gesellschaft
}

Roter Umschlag und zwölf Seiten Inhalt - die ersten Ausgaben der Schweizerischen Ärztezeitung im Mai 1920 präsentierten sich als Kampfblatt für die Standesinteressen der schweizerischen Ärzteschaft. Heute kommt die Tonalität der Artikel gemässigter daher, und das kämpferische Rot des Umschlags hat einem sonnigen Gelb Platz gemacht. Mit dem Wandel zum Diskussionsforum hat sich die SÄZ in diesen 100 Jahren eine zentrale Rolle im schweizerischen Gesundheitsbereich erarbeitet.

Die Lancierung der Schweizerischen Ärztezeitung 1920 war Teil grösserer Bewegungen bei der Kommunikation der FMH. Die bestehende Verbandszeitschrift, Correspondenz-Blatt für Schweizer Ärzte, wurde zur Schweizerischen Medizinischen Wochenzeitschrift (SMW), umgangssprachlich «Schwiwo», umbenannt. Der Namenswechsel ging mit einer Anpassung der inhaltlichen Ausrichtung einher. Die SMW erschien mehr im Stil eines «Medical Journal». Die neue Schweizerische Ärztezeitung für Standesfragen deckte hingegen hauptsächlich standes- und gesundheitspolitische Themen ab. Damit schuf sich die FMH ein zeittypisches Instrument, um den damaligen Kampf der Ärzte für einen starken und autonomen Berufsstand nicht nur auf dem politischen Parkett, sondern auch innerhalb der Ärzteschaft offensiv vertreten zu können.

\section{Aus Rot wird Gelb}

Richtig klappte dieses Vorhaben aufgrund von Unstimmigkeiten erst im zweiten Anlauf. So war bei den ersten Ausgaben die Ärztegesellschaft des Kantons Bern federführend. Ab Oktober übernahm dann der Zentralvorstand der FMH die redaktionelle Leitung. Während die «Schwiwo», wie schon das Correspondenz-Blatt, weiterhin in Basel beim Schwabe Verlag herausgegeben wurde, erschien die Schweizerische Ärztezeitung für Standesfragen (SÄZ) beim Berner Ernst Bircher Verlag, dem späteren Hans Huber Verlag. Der initial kämpferisch rote Umschlag der Zeitschrift wechselte im Jahr 1929 zu einem dezenten Gelbton - die SÄZ war als «Gelbes Heftli» geboren.

In der zweiten Jahrhunderthälfte entwickelte sich die $\mathrm{SÄZ}$ schrittweise von einem Verbandsorgan zu einer Informations- und einer Diskussionsplattform der Schweizer Ärzte- und später auch der Ärztinnenschaft. Immer mehr stand die Zeitschrift selbstbewusst neben und nicht unter der «Schwiwo». Das Format wurde grösser, das Gelb knalliger, der Umschlag glänzender, und der Umfang nahm auf über 40 Seiten zu. Dafür wurde der Titel der neuen Ausrichtung angepasst: Die «Standesfragen» wurden gestrichen.

\section{Gepflegtes Infotainment statt Einheitsbrei}

Mit den Jahrzehnten war die schweizerische Ärzteschaft vielgestaltiger geworden. In den 1990er Jahren wuchs so bei einigen innovationsfreudigen FMH-Vertretern die Unzufriedenheit über die «sehr beschränkten Gestaltungsmöglichkeiten» des Quasi-«Amtsblatts». Die FMH forderte mehrere Verlage auf, Konzepte für einen Relaunch der verschiedenen Publikationsorgane auszuarbeiten. Die Wahl fiel schliesslich auf den Schwabe Verlag. Eine gemeinsame Arbeitsgruppe entwickelte die verschiedenen Ideen weiter. Statt eines «Einheitsbreis» solle in der SÄZ künftig die «Pluralität gesundheitspolitischer Meinungen» abgebildet werden. Man wollte Kommentare, Seitenblicke in andere Fachgebiete, futuristische Spekulationen neben historischer Retrospektive, Personalia, Satirisches - eine gelegentlich als Infotainment bezeichnete Umgebung, wie es der damalige FMH-Präsident Hans Heinrich Brunner beschrieb.

\section{Aus zwei mach drei}

Für den Relaunch der SÄZ gründete die FMH zusammen mit dem Schwabe Verlag (heute die Petri Holding AG) den Schweizerischen Ärzteverlag EMH (Editores Medicorum Helveticorum) mit Sitz in Basel. Die Mehrheit der Unternehmensanteile hält die FMH. Viele der Gründer, wie beispielsweise Ludwig T. Heuss oder Natalie Marty, wurden von ambitionierten Planern zu engagierten Machern der verschiedenen Medienprodukte und sind es bis heute geblieben.

Das Alleinstellungsmerkmal der «neuen SÄZ» aus dem Hause EMH war und ist die redaktionelle Unabhängigkeit. Diese redaktionelle Freiheit trägt massgeblich zum Ansehen der SÄZ als liberaler Diskussionsplattform bei. So begutachtet eine divers zusammengesetzte Redaktionskommission während des Review-Prozesses die eingereichten Artikel und entscheidet gemeinsam über deren Annahme bzw. Ablehnung. 
Um die Bedürfnisse der Leserschaft noch besser abdecken zu können, entschloss sich die Verlagsleitung 2001, die Themenbereiche der SÄZ und der SMW auf drei Zeitschriften aufzuteilen: Neben der gesundheitspolitisch orientierten Ärztezeitung entstanden eine Zeitschrift für aktuelle Forschungsergebnisse unter dem Namen Swiss Medical Weekly und eine reine Fortbildungszeitschrift, das Swiss Medical Forum.

\section{Dualer Aufbau als Erfolgsrezept}

Dem Anspruch, eine Plattform für alle schweizerischen Ärztinnen und Ärzte zu sein, kam die SÄZ mit einem neuen, konsequenteren Einbezug der Romands entgegen. Seit 2006 erscheint mit dem Bulletin des médecins suisses eine mehrheitlich französischsprachige Druckausgabe der $\mathrm{SÄZ}$.

Im selben Jahr kam es zu einem weiteren bemerkenswerten Entscheid: der jetzt konsequenten dualen Aufteilung des Inhalts. So nutzt die FMH den vorderen, blau markierten Teil zur Publikation ihrer eigenen Beiträge. Im hinteren Teil finden Artikel verschiedenster Autorinnen und Autoren Platz. Entsprechend vielfältig ist das Themenspektrum. Die dabei zum Ausdruck gebrachten unterschiedlichen (gesundheits-)politischen, ethischen und gesellschaftlichen Anschauungen sind ein klares Indiz dafür, dass der duale Aufbau der SÄZ ein funktionierendes Modell darstellt, um das plurale Schweizer Gesundheitswesen unter dem Dach eines grossen Berufsverbandes abzubilden.

In den letzten Jahren beeinflusste die Digitalisierung die Publikationsformen massgeblich. So baute der EMHVerlag den Online-Auftritt der verschiedenen Zeitschriften stetig aus. Zudem ist die SÄZ auf Social-Media-Kanälen wie Facebook und Twitter präsent.

Vor hundert Jahren als Kampfblatt entstanden, wurde die SÄZ als gesundheitspolitisches Forum zum Flaggschiff der FMH. Auch wenn sich das Leserverhalten und die Medienlandschaft kontinuierlich verändert haben, konnte die SÄZ ihre Stellung als gesundheitspolitische Zeitschrift mit schweizweiter Bedeutung halten - nicht zuletzt dank unzähligen engagierten Autorinnen, Autoren und Mitarbeitenden.

\section{Bildnachweis}

Grafik: lötscher visuelle kommunikation

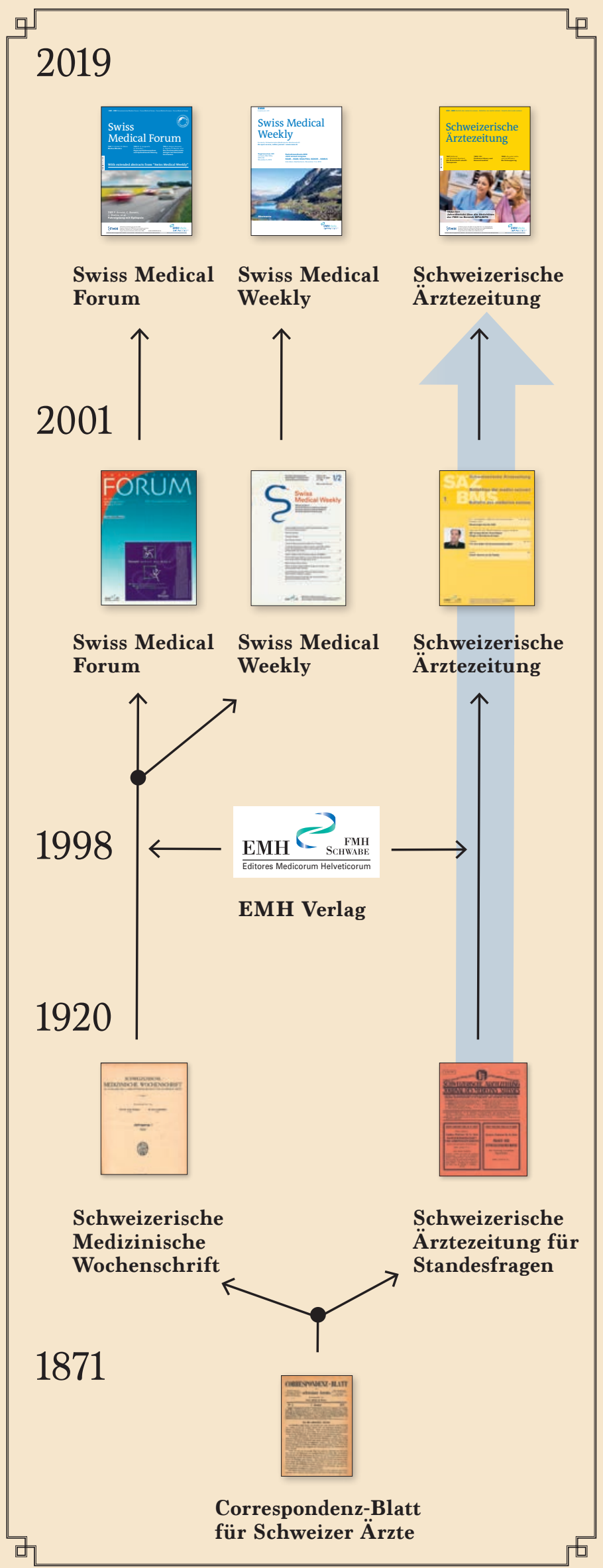

